\title{
Habitability of super-Earths: Gliese 581c \& 581d
}

\author{
W. von Bloh ${ }^{1}$, C. Bounama ${ }^{1}$, M. Cuntz ${ }^{2}$ and S. Franck ${ }^{1}$ \\ ${ }^{1}$ Potsdam Institute for Climate Impact Research, \\ P.O. Box 601203, Potsdam, Germany, \\ email: bloh@pik-potsdam.de, bounama@pik-potsdam.de, franck@pik-potsdam.de \\ ${ }^{2}$ University of Texas at Arlington, \\ P.O. Box 19059, Arlington, TX 76019, USA \\ email: cuntz@uta.edu
}

\begin{abstract}
The unexpected diversity of exoplanets includes a growing number of super-Earth planets, i.e., exoplanets with masses smaller than 10 Earth masses. Unlike the larger exoplanets previously found, these smaller planets are more likely to have similar chemical and mineralogical composition to the Earth. We present a thermal evolution model for super-Earth planets to identify the sources and sinks of atmospheric carbon dioxide. The photosynthesis-sustaining habitable zone ( $\mathrm{pHZ}$ ) is determined by the limits of biological productivity on the planetary surface. We apply our model to calculate the habitability of the two super-Earths in the Gliese 581 system. The super-Earth Gl 581c is clearly outside the pHZ, while Gl 581d is at the outer edge of the pHZ. Therefore, it could at least harbor some primitive forms of life.
\end{abstract}

Keywords. Astrobiology, planetary systems, stars: individual (Gliese 581)

\section{Introduction}

Very recently, Udry et al. (2007) announced the detection of two super-Earth planets in the Gliese 581 system; namely, Gl 581c with a mass of $5.06 M_{\oplus}$ and a semi-major axis of $0.073 \mathrm{AU}$, and Gl 581d with $8.3 M_{\oplus}$ and 0.25 AU. Both mass estimates are minimum masses uncorrected for $\sin i$. The luminosity of Gl 581 can be estimated as $L=0.013 \pm 0.002 L_{\odot}$ with a stellar temperature of $T_{e}=3480 \mathrm{~K}$ and a stellar age of at least 2 Gyr. The main question is whether any of the two super-Earths around Gl 581 can harbor life, i.e., that any of the planets lie within the habitable zone (HZ). Typically, stellar HZs are defined as regions around the central star, where the physical conditions are favorable for liquid water to exist at the planet's surface for a period of time long enough for biological evolution to occur (Kasting et al. 1993).

In the following, we adopt a definition of the HZ previously used by Franck et al. (2000). Here habitability does not just depend on the parameters of the central star, but also on the properties of the planet. In particular, habitability is linked to the photosynthetic activity of the planet, which in turn depends on the planetary atmospheric $\mathrm{CO}_{2}$ concentration, and is thus strongly influenced by the planetary dynamics. We call this definition the photosynthesis-sustaining habitable zone $(\mathrm{pHZ})$. In principle, this leads to additional spatial and temporal limitations of habitability.

\section{Estimating the Habitability of a Super-Earth}

To assess the habitability of a super-Earth, i.e., a rocky planet smaller than 10 Earth masses (Valencia et al. 2006), an Earth-system model is applied to calculate the evolution of the temperature and atmospheric $\mathrm{CO}_{2}$ concentration. The numerical model couples 
the stellar luminosity, the silicate-rock weathering rate, and the global energy balance to obtain estimates of the partial pressure of atmospheric carbon dioxide $P_{\mathrm{CO}_{2}}$, the mean global surface temperature $T_{\text {surf }}$, and the biological productivity $\Pi$ as a function of time $t$. The main point is the persistent balance between the $\mathrm{CO}_{2}$ sink in the atmosphereocean system and the metamorphic (plate-tectonic) sources. This is expressed through the dimensionless quantities

$$
f_{\mathrm{wr}}(t) \cdot f_{A}(t)=f_{\mathrm{sr}}(t),
$$

where $f_{\mathrm{wr}}(t)$ is the weathering rate, $f_{A}(t)$ is the continental area, and $f_{\mathrm{sr}}(t)$ is the spreading rate normalized by their present values of Earth. The connection between the stellar parameters and the planetary climate can be formulated by using a radiation balance equation. The evolution of the surface temperature is derived directly from the stellar luminosity, the distance to the central star and the geophysical forcing ratio $\left(\mathrm{GFR}:=f_{\mathrm{sr}} / f_{A}\right)$. For the investigation of a super-Earth under external forcing, we adopt a model planet with a prescribed continental area. The fraction of continental area to the total planetary surface is varied between 0.1 and 0.9 .

The thermal history and future of a super-Earth is determined by calculating the GFR values. Spreading rates can be derived from the mantle temperature. Assuming conservation of energy, the average mantle temperature $T_{m}$ is obtained as

$$
\frac{4}{3} \pi \rho c\left(R_{m}^{3}-R_{c}^{3}\right) \frac{d T_{m}}{d t}=-4 \pi R_{m}^{2} q_{m}+\frac{4}{3} \pi E(t)\left(R_{m}^{3}-R_{c}^{3}\right)
$$

where $\rho$ is the density, $c$ is the specific heat at constant pressure, $q_{m}$ is the heat flow from the mantle, $E$ is the energy production rate by decay of radiogenic heat sources in the mantle per unit volume, and $R_{m}$ and $R_{c}$ are the outer and inner radii of the mantle, respectively. The photosynthesis-sustaining $\mathrm{HZ}(\mathrm{pHZ})$ is defined as the spatial domain encompassing all distances $R$ from the central star where the biological productivity is greater than zero, i.e.,

$$
\mathrm{pHZ}:=\left\{R \mid \Pi\left(P_{\mathrm{CO}_{2}}(R, t), T_{\text {surf }}(R, t)\right)>0\right\} .
$$

In our model, biological productivity is considered to be solely a function of the surface temperature and the $\mathrm{CO}_{2}$ partial pressure in the atmosphere. Our parameterization yields zero productivity for $T_{\text {surf }} \leqslant 0^{\circ} \mathrm{C}$ or $T_{\text {surf }} \geqslant 100^{\circ} \mathrm{C}$ or $P_{\mathrm{CO}_{2}} \leqslant 10^{-5}$ bar (Franck et al. 2000). To calculate the spreading rates for a planet with several Earth masses, the planetary parameters have been adjusted following Valencia et al. (2006) as

$$
\frac{R_{p}}{R_{\oplus}}=\left(\frac{M}{M_{\oplus}}\right)^{0.27}
$$

where $R_{p}$ is the planetary radius and $M$ is the mass, with subscript $\oplus$ denoting Earth values. See von Bloh et al. (2007) for details and specified parameter values.

\section{Results and Discussion}

The habitable zone around Gl 581 for planets with five and eight Earth masses has been calculated for $L=0.013 L_{\odot}$. The results for Gl 581c and Gl 581d are shown in Fig. 1a,b. The simulations have been carried out for a maximum $\mathrm{CO}_{2}$ pressure of 5 bar (light colors) and 10 bar (dark colors) neglecting the cooling effect of $\mathrm{CO}_{2}$ clouds. The super-Earth planet Gl 581c is found to be clearly outside the habitable zone. On the other hand, one might expect that life had a chance to originate on Gl 581d because it is near the outer edge of the $\mathrm{pHZ}$, and for some combination of system parameters even 

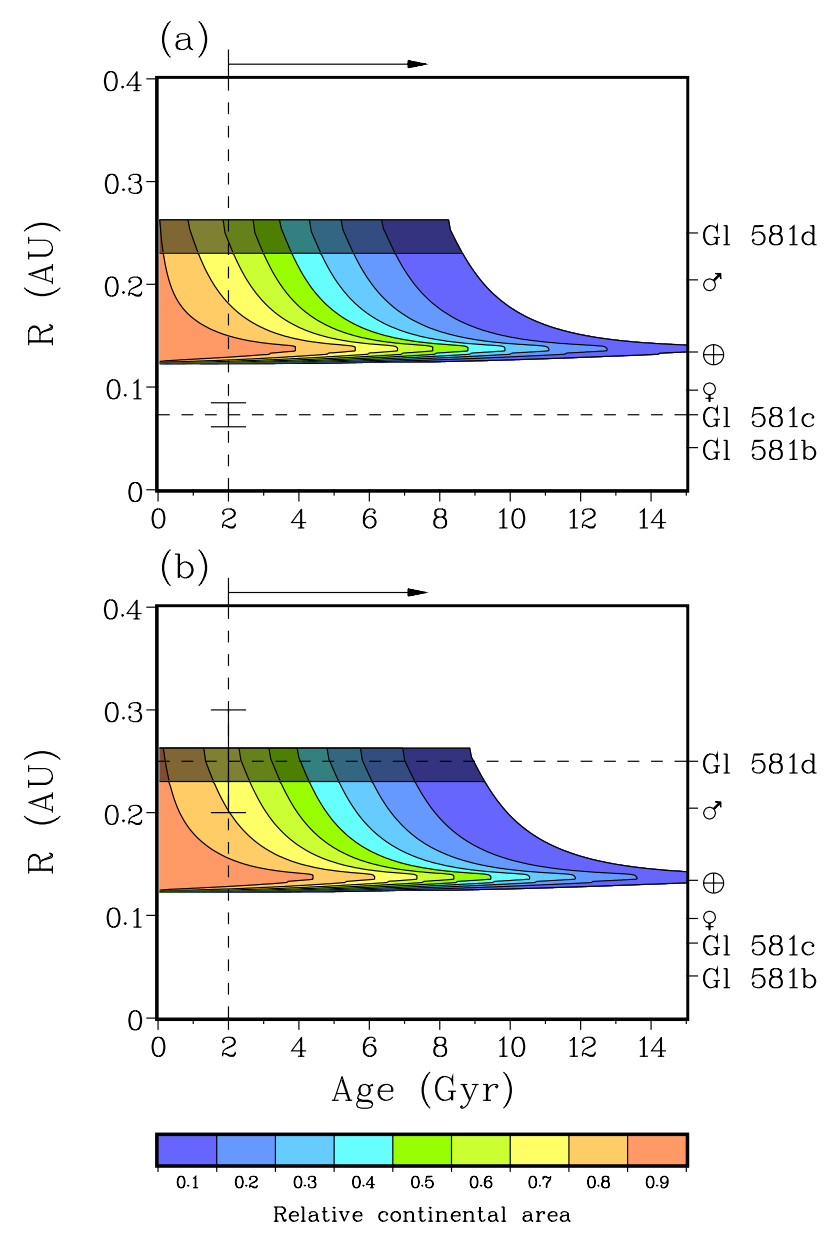

Figure 1. The pHz zone of Gl 581 for super-Earth Gl 581c (a) and Gl 581d (b) with a relative continental area varied from 0.1 to 0.9 and a fixed stellar luminosity of $0.013 L \odot$. The results are given as a function of planetary age. The light colors correspond to a maximum $\mathrm{CO}_{2}$ pressure of 5 bar, whereas the dark colors correspond to 10 bar. For comparison, the positions of Venus, Earth and Mars are shown scaled to the luminosity of Gl 581.

inside the pHZ (von Bloh et al. 2007). A planet of eight Earth masses has more volatiles than an Earth-size planet and can build up a sufficiently dense atmosphere to prevent it from freezing out due to tidal locking. Planets inside the habitable zone around M stars may be tidally locked, which however does not necessarily thwart habitability (Tarter et al. 2007). The modestly eccentric orbit of Gl 581d ( $e=0.2 \pm 0.1$ ) further supports habitability, even if the maximum $\mathrm{CO}_{2}$ pressure is assumed to be as low as 5 bar. The appearance of complex life, however, is unlikely due to the rather adverse environmental conditions. To get an ultimate answer to the profound question of life on Gl 581d, we have to await the TPF/Darwin missions. They will allow for the first time the detection of biomarkers in the atmospheres of the two super-Earths around Gl 581. 


\section{References}

Franck, S., Block, A., von Bloh, W., Bounama, C., Schellnhuber, H.-J., \& Svirezhev, Y. 2000, Tellus, 52B, 94

Kasting, J. F., Whitmire, D. P., \& Reynolds, R. T. 1993, Icarus, 101, 108

Tarter, J. C., et al. 2007, Astrobiology, 7, 30

Udry, S., et al. 2007, A\&A, 469, L43

Valencia, D., O'Connell, R. J., \& Sasselov, D. 2006, Icarus, 181, 545

Von Bloh, W., Bounama, C., Cuntz, M., \& Franck, S. 2007, A $\& A, 476,1365$ 